\title{
One visit apexification using a novel dental material Biodentine - A Case report
}

\author{
Dr. Akilan Balasubrahmaniam ${ }^{1}$, Dr. Sabna B S², Dr. M. Ramesh Kumar ${ }^{3}$, Dr. \\ Jayasree. $S^{4}$ \\ ${ }^{1,2}$ (Junior Residents,Departmentof Conservative Dentistry and Endodontics) \\ ${ }^{3}$ (Professor and Head,Department of Conservative Dentistry and Endodontics) \\ ${ }^{4}$ (Associate Professor, Department of Conservative Dentistry and Endodontics
}

\begin{abstract}
The management of a nonvital tooth with open apex consists of either the induction of a natural barrier or the creation of a artificial apical barrier. Since the conventional apexification using calcium hydroxide has certain drawbacks recent approach is to form an artificial apical plug. In addition to the current gold standard, MTA, a large number of novel materials have been studied for the purpose. This article describes the successful management of a traumatized and necrotic permanent maxillary central incisor with an open apex using artificial apical barrier technique with a novel material, Biodentine
\end{abstract}

Key words: Artificial apical plug, Biodentine, One visit apexification

\section{Introduction}

Proper assessment of the affected tooth is critical in determining an accurate diagnosis and prescribing the appropriate treatment plan. The complete formation of root and closure of the apical foramen occurs up to 3 years after the eruption1. Traumatic dental injuries to young permanent teeth during this period may cause pulpal inflammation or necrosis and subsequent incomplete development of the dentinal walls and root apices. These teeth present wide dentinal tubules that allow the penetration of bacteria and their irritants.

The management of a nonvital tooth with open apex consists of the induction of a natural or artificial apical barrier which can act as a stop for the obturating material. Apexification is defined as 'a method to induce a calcified barrier in a root with an open apex or the continued apical development of an incomplete root in teeth with necrotic pulp'2. Conventional apexification procedure is inducing the formation of an apical barrier while the recent approach is to form an artificial apical barrier by the placement of an apical plug.

A number of materials have been used for creating an artificial apical barrier including Resorbable ceramic, Tricalcium phosphate, Calcium hydroxide, Surgicel/amalgam, Freeze-dried bone or dentin. Among the various materials mentioned, Mineral Trioxide Aggregate (MTA) is currently considered as one of the most promising materials because of its superior biocompatibility and less cytotoxicity due to its alkaline $\mathrm{pH}$ and presence of calcium and phosphate ions resulting in capacity to attract blastic cells and promote favorable conditions for cementum deposition3. However MTA has certain disadvantages like high solubility4, prolonged setting time of approximately 2 hours and 45 minutes 5 as well as the difficult handling characteristics.

Recently a novel material named biodentine has been introduced by Septodont in September 2010. Biodentine is similar to MTA in its basic composition with the addition of setting accelerators which is calcium chloride not only results in fast setting but also improves the handling properties and strength. Biodentine is superior to MTA like its consistency is better suited to the clinical use, ensures a better handling and safety, does not require a two-step obturation and as the setting is faster, there is a lower risk of bacterial contamination6.

This article describes the successful management of a symptomatic, traumatized, necrotic permanent maxillary central incisor with wide open apex using biodentine as an artificial apical barrier.

\section{Case Report}

A 43 year old male patient, reported to the Department of Conservative Dentistry and Endodontics, Govt Dental College Calicut with a chief compliant dull pain and swelling in relation to upper front tooth region for the past 2 months. The patient had a history of trauma to the permanent maxillary left central incisor at the age of 8 yrs. Medical history was non contributory and the clinical examination revealed presence of sinus tract in relation to discoloured tooth [fig1]. The concerned tooth did not respond to both electric and thermal pulp sensibility test. Detailed radiographic examination revealed a large blunderbuss canal with associated periapical lesion in relation to involved tooth [fig2]. Based on the history and the radiographic findings, a provisional diagnosis of chronic periapical abscess was made in relation to immature necrotic maxillary left central incisor Informed consent was obtained from the patient. After rubber dam application under local anaesthesia access opening was done. Working length was established. Biomechanical preparation was done using No 60 stainless 
steel $\mathrm{k}$ file (Dentsply, Maillefer) under copious irrigation with $5.25 \% \mathrm{NaOCl}$. Calcium hydroxide intracanal medicament (RC Cal, Prime Dental) was placed for 2 weeks. The patient was recalled after 2 weeks and the involved tooth was found to be asymptomatic. The access cavity was reopened, copious irrigation was done with $\mathrm{NaOCl}$ solution,the root canal was dried with sterile paper points.

Biodentine was mixed according to manufacturer's protocol and it was placed with a plugger until a thickness of $5 \mathrm{~mm}$ [fig3]. A sterile cotton pellet was placed in the canal for $15 \mathrm{~min}$ and the root canal was obturated by thermo plasticized Gutta-percha technique (Obtura - Spartan) [fig4]. The access cavity was then sealed with the composite restoration and the discolored tooth was restored by porcelain fused to metal crown [fig5, 6]. Follow up examinations were carried out at 1month, 3 months, 6 months [fig7] and 1 year after apexification [fig8]. During follow up periods the involved tooth was asymptomatic and the Post operative radiographs taken at 6 months and 1 year showed remarkable healing of the osseous lesion.

\section{Discussion}

Immature permanent teeth pose special challenges during endodontic procedures not only because of the wide-open root apex but also because of the thin dentin walls7. Induction of apical healing, regardless of the material used, takes at least 3-4 months and requires multiple appointments8. Patient compliance with this regimen may be poor and many fail to return for scheduled visits. The temporary seal may fail resulting in reinfection and failure of treatment.

Morse et al define one-visit apexification as the non-surgical condensation of a biocompatible material into the apical end of the root canal9. The rationale is to establish an apical stop that would enable the root canal to be filled immediately. There is no attempt at root end closure. Rather an artificial apical stop is created. The advantages of using an apical plug include decreased number of patients visit, more predictable apical barrier formation, and reduced need for follow-up appointments.

Biodentine with Active Biosilicate Technology was introduced by Septodont in September of 2010, as "a new class of dental material which could conciliate high mechanical properties with excellent biocompatibility, as well as bioactive behaviour" 10

Usually calcium silicate cements have setting times in the range of several hours, which is too long in most of the protocols in clinical practice. Adding calcium chloride to the liquid component accelerates the system. Finally, the decrease of the liquid content in the system decreases the setting time to harden within 9 to 12 minutes 11 .

Biodentine has the capacity to continue improving in strength with time over several days until reaching $300 \mathrm{MPa}$ after one month. This maturation process can be related to the decrease of porosity with time12. Studies on the biological effects of Biodentine on immortalized murine pulp cells concluded that Biodentine shows apatite formation after immersion in phosphate solution indicative of its bioactivity. The deposition of apatite structures might increase the marginal sealing of the material13.

\section{Conclusion}

The use of Biodentine in creating an artificial apical barrier explores a new paradigm in the field of apexification. The excellent biocompatibility and shorter setting time of Biodentine makes it a promising material for one visit apexification. However long term clinical trials with follow up are further required

\section{References}

[1]. Bhasker SN. Orban's oral histology and embryology, 11th edn. St. Louis: Mosby-Year Book; 1991

[2]. American Association of Endodontists. Glossary of endodontitc terms, 7 th edn. Chicago: American Association of Endodontists; 2003

[3]. Kubasad GC, Ghivari SB. Apexification with apical plug of MTA-report of cases. Arch Oral Sci Res 2011

[4]. Fridland M \& Rosado R (2003) Mineral Trioxide aggregate (MTA) solubility and porosity with different water-to-powder ratios. Journal of Endodontics

[5]. Torabinejad M, Hong C, McDonald F \& Pitt Ford T (1995) Physical and chemical properties of a new root-end filling material Journal of Endodontics 21(7) 349-353

[6]. Pawar AM, Kokate SR, Shah RA. Management of a large periapical lesion using Biodentine TM as retrograde restoration with eighteen months evident follow up. J Conserv Dent 2013;16:573-5

[7]. Apexification: a review. Dent Traumatology 2005; 21: 1-8.

[8]. Treatment Options: Apexogenesis and Apexification; J Endod 2013;39:S26-S29

[9]. Morse DR, O'Larnic J, Yesilsoy C. Apexification: review of the literature. Quintessence Int 1990;21:589-98

[10]. Septodont Biodentine ${ }^{\mathrm{TM}}$ scientific file, 2010)

[11]. Laurent P, Camps J, About I. Biodentine(TM) induces TGF-beta1 release from human pulp cells and early dental pulp mineralization. Int Endod J. 2012 May 45(5):439-48.

[12]. Camilleri J, Sorrentino F, Damidot D. Investigation of the hydration and bioactivity of radiopacified tricalcium silicate cement, Biodentine and MTA Angelus. Dent Mater. 2013 May;29(5):580-93.

[13]. Zanini M, Sautier JM, Berdal A, Simon S. Biodentine induces immortalized murine pulp cell differentiation into odontoblast-like cells and stimulates biomineralization. J Endod. 2012 Sep 

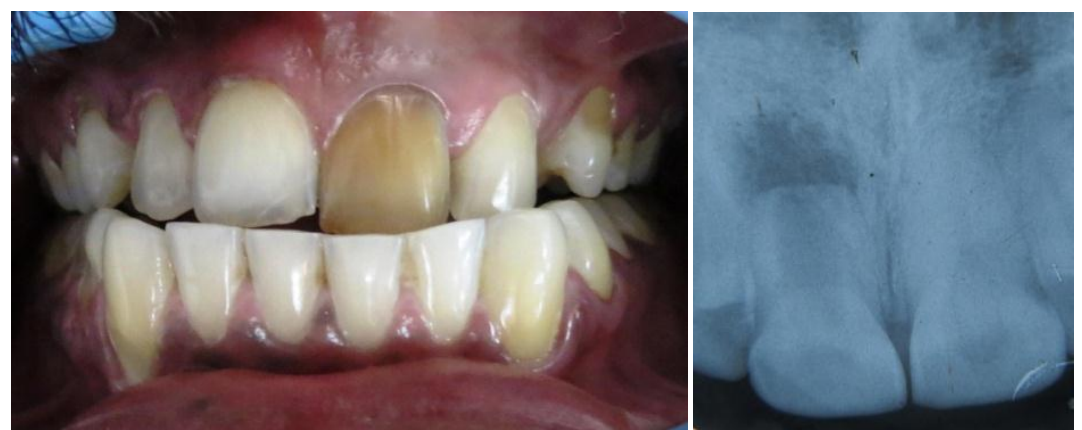

Figure1-preoperative clinical view

Figure 2-preoperative radiograph
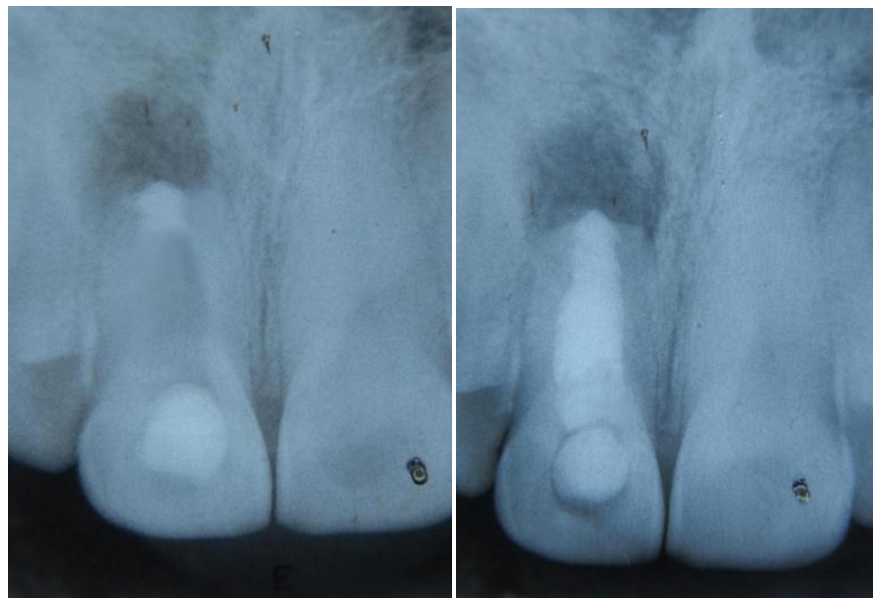

Figure3- Biodentine placement

figure4- obturation with gutta percha

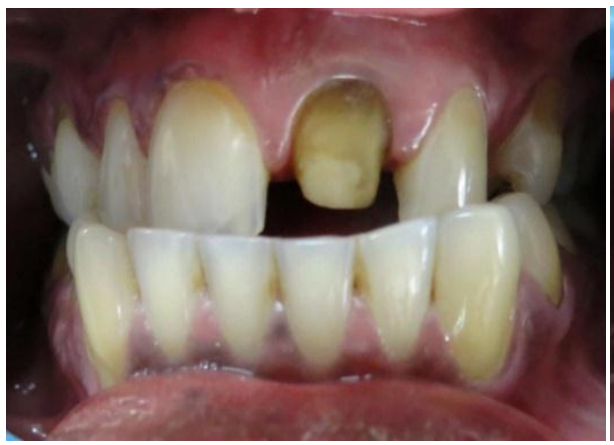

Figure5 - Tooth preparation

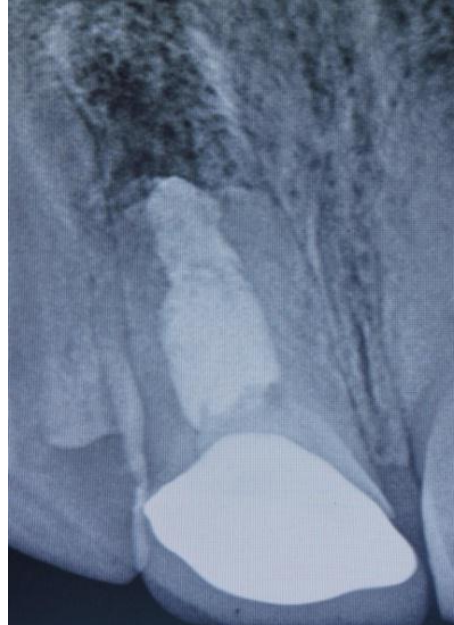

Figure7- 6 month follow up

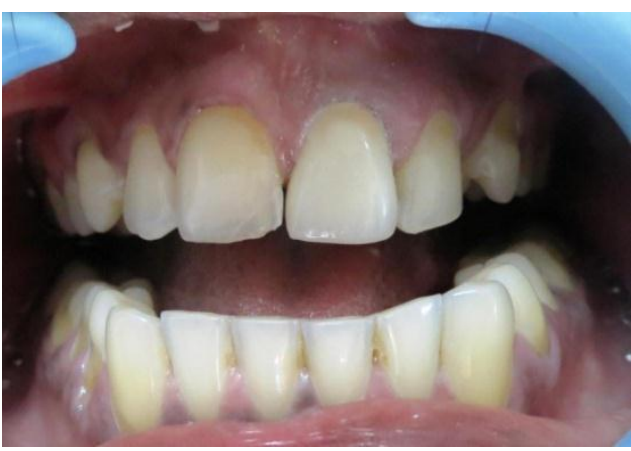

Figure6- PFM crown placement

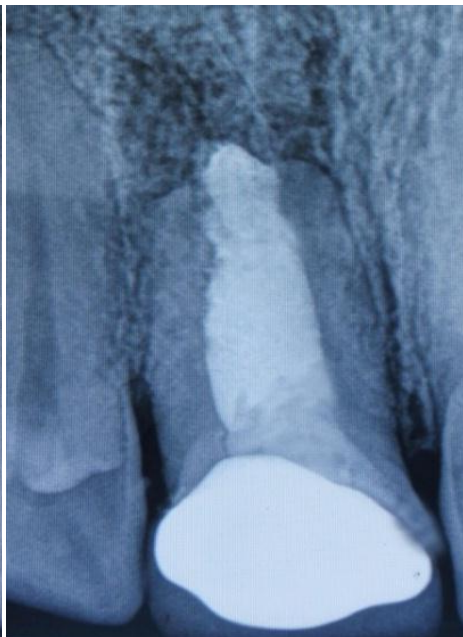

Figure8- 1 year follow up 